'G V Escota, ${ }^{2}$ T Taniguchi, 'B P Stoner, ${ }^{1} \mathrm{~N}$ F Onen. 'Washington University School of Medicine, Division of Infectious Diseases, Saint Louis, MO, United States; ${ }^{2}$ Chiba University Graduate School of Medicine, Department of Developmental Genetics, Chiba, Japan

Background Recent data suggest sexual transmission of hepatitis $\mathrm{C}$ virus (HCV). However, data on the association between HCV and sexually transmitted disease (STD) prevalence are limited.

Methods This was a retrospective cohort study of treatment-naïve HIV-infected adults $\geq 18$ years first engaging at Washington University HIV Clinic from 2001 to 2009, who had routine STD and HCV antibody testing done. Gonorrhea, chlamydia, syphilis, and HCV cases were defined by positive urine nucleic acid amplification test for Neisseria gonorrhoeae, Chlamydia trachomatis, reactive serum rapid plasma reagin, and positive HCV antibody, respectively. Associations with HCV and STD using $\chi 2$, Student's t, and Wilcoxon tests were determined.

Discussion Of 926 subjects (median age 32 years, 70\% African American, 44\% heterosexual, 42\% men-who-have-sex-with-men [MSM], 4\% injection drug users [IDU]), 8\% had HCV (range $5-11 \%$ /year). Baseline STD was prevalent in $27 \%$ (18-34\%/year). The prevalence of gonorrhoea, chlamydia and syphilis were $12 \%$ (7-21\%/year), 12\% (6-17\%/year) and 10\% (5-16\%/year), respectively. Subjects with HCV were older (42 years, interquartile range [IOR 38-48] versus 31 years, [IOR 24-40]) $(p<0.001)$ and more likely to report past IDU $(30 \%$ versus $2 \%)(p<0.001)$ than those without. Male subjects with HCV were less likely to be MSM (28\% vs $66 \%)(p<0.001)$ and $36 \%$ of subjects with HCV were heterosexuals without past IDU. Subjects with HCV were less likely to have STD $(17 \%$ vs $28 \%, p=0.06)$, although this finding did not reach statistical significance. Furthermore, the number and type of STDs at presentation were not associated with prevalent HCV.

Conclusion Hepatitis C was prevalent in approximately 1 in 10 persons engaging in HIV outpatient care over nine years. A high prevalence of HCV among heterosexuals without past IDU suggests a possible role for sexual transmission of HCV not reflected by STD prevalence. Continued universal HCV screening among HIVinfected adults is imperative.

\section{P3.241 EPIDEMIOLOGY OF SEXUALLY TRANSMITTED INFECTIONS IN EUROPE 1990-2011}

doi:10.1136/sextrans-2013-051184.0698

G Spiteri, M van de Laar. European Centre for Disease Prevention and Control, Stockholm, Sweden

Background STI are a significant cause of morbidity in Europe, particularly among young adults and men who have sex with men (MSM). Monitoring the spread of STI across key populations allows development of targeted prevention interventions.

Methods Surveillance for chlamydia, gonorrhoea and syphilis in the European Union (EU/EEA) is coordinated by the European Centre for Disease Prevention and Control since 2009. Data reported by Member States covering the period 1990-2011 were analysed.

Results In 2011, 346911 cases of chlamydia (rate: 175 per 100000 population) were reported compared to 39179 cases of gonorrhoea (12.6/100000) and 19798 cases of syphilis (4.9/100000). Chlamydia was more frequently reported among women (male-to-female ratio: 0.7 ) in contrast to gonorrhoea and syphilis (male-to-female ratios: 2.7 and 3.9 respectively). The highest age and gender-specific rates were observed among 15-19 year old females for chlamydia (1748); 20-24 year-old males for gonorrhoea (71) and 25-24 year-old males for syphilis (16.1). Young adults (15-24 years) accounted for $73 \%$ of chlamydia, $42 \%$ of gonorrhoea and $16 \%$ of syphilis cases. Transmission among MSM was more frequently reported for syphilis (42\%) and gonorrhoea (33\%) cases than for chlamydia (5\%). HIV co-infection was reported among $11 \%$ of gonorrhoea and $28 \%$ of syphilis cases. Rates of chlamydia have increased by $133 \%$ between 2000 and 2011 among countries reporting consistently. Gonorrhoea rates have increased by $27 \%$ since 2008 and syphilis rates increased by $10 \%$ in 2011.

Conclusion Surveillance data shows diversity in reported rates and trends across the EU/EEA. Increasing rates of chlamydia reflect strengthened case detection and improved diagnostics. Young adults constitute a large proportion of cases of chlamydia and gonorrhoea. Transmission among MSM accounts for the majority of syphilis and, increasingly, gonorrhoea spread. Both key populations need to be better targeted through specific prevention and control measures.

\section{P3.242 WITHDRAWN BY AUTHOR}

\section{P3.243 INFLUENCE OF SCALE AND ZONE ON SYPHILIS TREND INTERPRETATION}

doi:10.1136/sextrans-2013-051184.0699

1.2V Escamilla, ${ }^{3} \mathrm{~K}$ Hampton, ${ }^{3,4,1 \mathrm{P}}$ A Leone, ${ }^{1,3} \mathrm{~W}$ C Miller. 'Division of Infectious Diseases, Department of Medicine, University of North Carolina, Chapel Hill, NC, United States; ${ }^{2}$ Carolina Population Center, University of North Carolina, Chapel Hill, NC, United States; ${ }^{3}$ Department of Epidemiology, Gillings School of Global Public Health, University of North Carolina, Chapel Hill, NC, United States; ${ }^{4}$ HIV/STD Prevention and Care Branch, North Carolina Division of Public Health, Raleigh, NC, United States

Background We investigated spatial clustering of primary and secondary syphilis rates in North Carolina (2003-2010) using multiple scales and geographical boundaries. We examined the influence of changes in scale and boundary on identification of spatial clusters using two cluster detection methods, local Moran's I and Kulldorff's space scan statistic.

Methods We used two cluster detection methods: (1) local Moran's I with empirical Bayes (EB) standardised rate statistic and (2) Kulldorff's space scan statistic using a variable size moving circular window. We evaluated three geographic zones with decreasing boundary area, North Carolina, Piedmont region, and Mecklenburg County, at two spatial scales (census tract, census block group). We report results for Mecklenburg County.

Results Using local Moran's I, block group clusters were in the same location as tract clusters, but were more concentrated. Median rates were higher among block group clusters compared with tract clusters. As boundary areas decreased, some clusters in peripheral tracts and block groups were lost. High rate block groups were more likely to persist, while some high rate peripheral tracts were lost.

With Kuldorff's scan statistic, block group clusters were more concentrated than clusters in census tracts. Reducing boundary areas had little effect on census tract clusters detected using Kuldorff's scan statistic. Cluster size decreased significantly when the boundary was restricted to Mecklenburg County. The reduction in cluster size reflected loss of a few high rate block groups peripherally and many block groups with a rate of zero.

Clusters detected using local Moran's I and Kuldorff's scan statistic overlapped, but Kuldorff's scan clusters were much larger with a high proportion of zero rate tracts/block groups.

Conclusion In efforts to understand STI epidemiology spatially, investigators must carefully consider the spatial scale, geographical area of interest, and cluster detection approach.

\section{P3.244 PREVALENCE OF SEXUALLY TRANSMITTED INFECTIONS IN MULTIPLE SAMPLE TYPES COLLECTED FROM HIV-1 POSITIVE MEN}

doi:10.1136/sextrans-2013-051184.0700

${ }^{1} \mathrm{~F}$ Zhang, ${ }^{2} \mathrm{~S}$ R Morris, ${ }^{3} \mathrm{~J}$ P McGowan, ${ }^{2} \mathrm{~J}$ Milam, ${ }^{4} \mathrm{M}$ Dube, ${ }^{5} \mathrm{E} S \mathrm{Daar},{ }^{3} \mathrm{D}$ Dubson, ${ }^{6} \mathrm{C} S$ Hill, ${ }^{2} \mathrm{R}$ H Haubrich, ${ }^{1,7}$ C C Ginocchio. ${ }^{1}$ North Shore-LIJ Health System Laboratories, Lake Success, NY, United States; 'niversity of California, San Diego, CA, United States, 